\title{
Oxidative stress, new insight in emergency toxicology; the role of 8-hydroxy-2-deoxyguanosine; a toxicologist viewpoint
}

\author{
Farzad Gheshlaghi ${ }^{1,2^{*}}$, Shayan Gheshlaghi ${ }^{3}$ \\ ${ }^{1}$ Department of Clinical Toxicology, School of Medicine, Isfahan University of Medical Sciences, Isfahan, Iran \\ ${ }^{2}$ Isfahan Clinical Toxicology Research Center, School of Medicine, Isfahan University of Medical Sciences, Isfahan, Iran \\ ${ }^{3}$ School of Medicine, Najaf Abad Branch, Islamic Azad University, Najaf Abad, Iran
}

\section{A R T I C L E IN F O}

Article Type:

Epidemiology and Prevention

\section{Article History:}

Received: 7 January 2018

Accepted: 6 February 2018

Published online: 10 February 2018

\section{Keywords:}

Oxidative stress, Reactive

oxygen species, 8-hydroxy-2-

deoxyguanosine, oxidative damage,

Emergency toxicology

\section{Implication for health policy/practice/research/medical education:}

In contrast to numerous studies on the function of oxidative stress including hypertension, diabetes mellitus, ischemic diseases, atherosclerosis and malignancies, limited studies have been done on the function of oxidative stress in determining the severity of acute and fatal poisoning as well as the therapies.

Please cite this paper as: Gheshlaghi F, Gheshlaghi Sh. Oxidative stress, new insight in emergency toxicology; the role of 8-hydroxy-2-deoxyguanosine; a toxicologist viewpoint. J Renal Inj Prev. 2018;7(3):119-120. doi: 10.15171/jrip.2018.28.
$\mathrm{O}$ xidative stress is recognized to be engaged in the pathogenesis of lifestyle-related illnesses, including malignancies, atherosclerosis, diabetes mellitus, hypertension, ischemic diseases, and environmental poisoning. Oxidative stress has been identified as a destructive event because oxygen free radicals strike biologic part of molecules such as proteins, lipids, and DNA molecule. Nevertheless, oxidative stress similarly has a beneficial role in physiologic adjustment and in the adjustment of intra-cellular signal transmission (1). For that reason, a more purposeful description of oxidative stress might be "a condition where oxidative strengths overdo the antioxidant forces because of deficit of the imbalance two side." The biomarkers that be able to be used to evaluate oxidative stress techniques, have been interesting in in-vivo studies because the precise weighting of such stress is essential for exploration of its role in life-style diseases as well as to assess the efficiency of treatment (1).

Studies showed that biomarker 8-hydroxy-2deoxyguanosine (8-OHdG) or 8-oxo-7,8-dihydro-
2deoxyguanosine(8-oxodG) has been a crucial indicator for assessing the impact of endogenous oxidative injury to DNA and as an element of beginning and endorsement of carcinogenesis process. Recently, 8-OHdG has been utilized extensively in many studies not only as a biomarker for quantify of endogen oxidative DNA damage but also as a risk factor for many illnesses as well as malignancies (2). From a clinical point of view, if a biomarker that reveals the magnitude of oxidative stress is accessible, such markers will be beneficial for physicians to achieve a vision into the pathological characteristics of a variety of diseases and evaluate the efficiency of treatment methods $(1,2)$.

In contrast to numerous studies on the function of oxidative stress comprising hypertension, atherosclerosis, ischemic diseases, diabetes mellitus, and malignancies, limited studies have been done on the function of oxidative stress and reactive oxygen radicals in determining the severity of acute and fatal poisoning as well as the therapies.

It seems that the determination of oxidative stress in the diagnosis of severity and response to therapy of acute poisoning can open up a new horizon. 


\section{Authors' contribution}

FG completed and reviewed the article. SHG searched the literature and prepared the primary draft. Both authors read and signed the final draft.

\section{Conflicts of interest}

The authors declared no competing interests.

\section{Ethical considerations}

Ethical issues (including plagiarism, data fabrication, double publication) have been completely observed by the authors.

\section{Funding/Support}

None.

\section{References}

1. Yoshikawa T, Naito Y. What is oxidative stress? JMAJ. 2002; 45:271-6.

2. Valavanidis A1, Vlachogianni T, Fiotakis C. 8-hydroxy-2' -deoxyguanosine (8-OHdG): A critical biomarker of oxidative stress and carcinogenesis. J Environ Sci Health C Environ Carcinog Ecotoxicol Rev. 2009;27:120-39.

Copyright (c) 2018 The Author(s); Published by Nickan Research Institute. This is an open-access article distributed under the terms of the Creative Commons Attribution License (http://creativecommons.org/licenses/by/4.0), which permits unrestricted use, distribution, and reproduction in any medium, provided the original work is properly cited. 